\title{
A Benchmark for Endoluminal Scene Segmentation of Colonoscopy Images
}

\author{
David Vázquez, ${ }^{1,2}$ Jorge Bernal, ${ }^{1}$ F. Javier Sánchez, ${ }^{1}$ Gloria Fernández-Esparrach, ${ }^{3}$ \\ Antonio M. López, ${ }^{1,2}$ Adriana Romero, ${ }^{2}$ Michal Drozdzal, ${ }^{4,5}$ and Aaron Courville ${ }^{2}$ \\ ${ }^{1}$ Computer Vision Center, Computer Science Department, Universitat Autonoma de Barcelona, Barcelona, Spain \\ ${ }^{2}$ Montreal Institute for Learning Algorithms, Université de Montréal, Montreal, QC, Canada \\ ${ }^{3}$ Endoscopy Unit, Gastroenterology Service, CIBERHED, IDIBAPS, Hospital Clinic, Universidad de Barcelona, Barcelona, Spain \\ ${ }^{4}$ École Polytechnique de Montréal, Montréal, QC, Canada \\ ${ }^{5}$ Imagia Inc., Montréal, QC, Canada
}

Correspondence should be addressed to David Vázquez; dvazquez@cvc.uab.es

Received 24 February 2017; Accepted 22 May 2017; Published 26 July 2017

Academic Editor: Junfeng Gao

Copyright ( 2017 David Vázquez et al. This is an open access article distributed under the Creative Commons Attribution License, which permits unrestricted use, distribution, and reproduction in any medium, provided the original work is properly cited.

Colorectal cancer (CRC) is the third cause of cancer death worldwide. Currently, the standard approach to reduce CRC-related mortality is to perform regular screening in search for polyps and colonoscopy is the screening tool of choice. The main limitations of this screening procedure are polyp miss rate and the inability to perform visual assessment of polyp malignancy. These drawbacks can be reduced by designing decision support systems (DSS) aiming to help clinicians in the different stages of the procedure by providing endoluminal scene segmentation. Thus, in this paper, we introduce an extended benchmark of colonoscopy image segmentation, with the hope of establishing a new strong benchmark for colonoscopy image analysis research. The proposed dataset consists of 4 relevant classes to inspect the endoluminal scene, targeting different clinical needs. Together with the dataset and taking advantage of advances in semantic segmentation literature, we provide new baselines by training standard fully convolutional networks (FCNs). We perform a comparative study to show that FCNs significantly outperform, without any further postprocessing, prior results in endoluminal scene segmentation, especially with respect to polyp segmentation and localization.

\section{Introduction}

Colorectal cancer (CRC) is the third cause of cancer death worldwide [1]. CRC arises from adenomatous polyps (adenomas) which are initially benign; however, over time, some of them can become malignant. Currently, the standard approach to reduce CRC-related mortality is to perform regular screening in search for polyps and colonoscopy is the screening tool of choice. During the examination, clinicians visually inspect the intestinal wall (see Figure 1(a) for an example of intestinal scene) in search of polyps. Once detected, they are resected and sent for histological analysis to determine their degree of malignancy and define the corresponding treatment the patient should undertake.
The main limitations of colonoscopy are its associated polyp miss rate (small/flat polyps or the ones hidden behind intestine folds can be missed [2]) and the fact that polyp's malignancy degree is only known after histological analysis. These drawbacks can be reduced by developing new colonoscopy modalities to improve visualization (e.g., highdefinition imaging, narrow-band imaging (NBI) [3], and magnification endoscopes [4]) and/or by developing decision support systems (DSS) aiming to help clinicians in the different stages of the procedure. A clinically useful DSS should be able to detect, segment, and assess the malignancy degree (e.g., by optical biopsy [5]) of polyps during the colonoscopy procedure, following a similar pipeline to the one shown in Figure 1(b). 


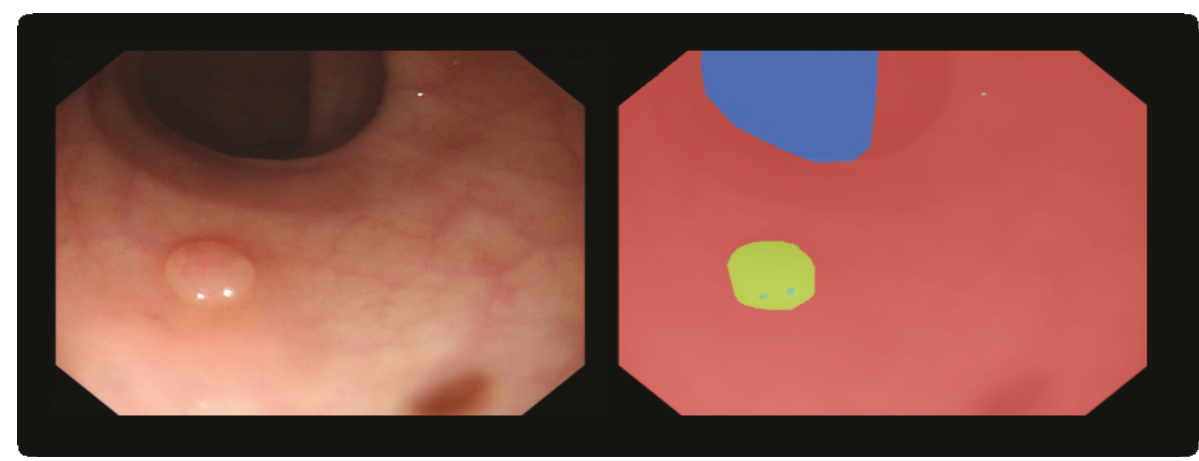

(a)
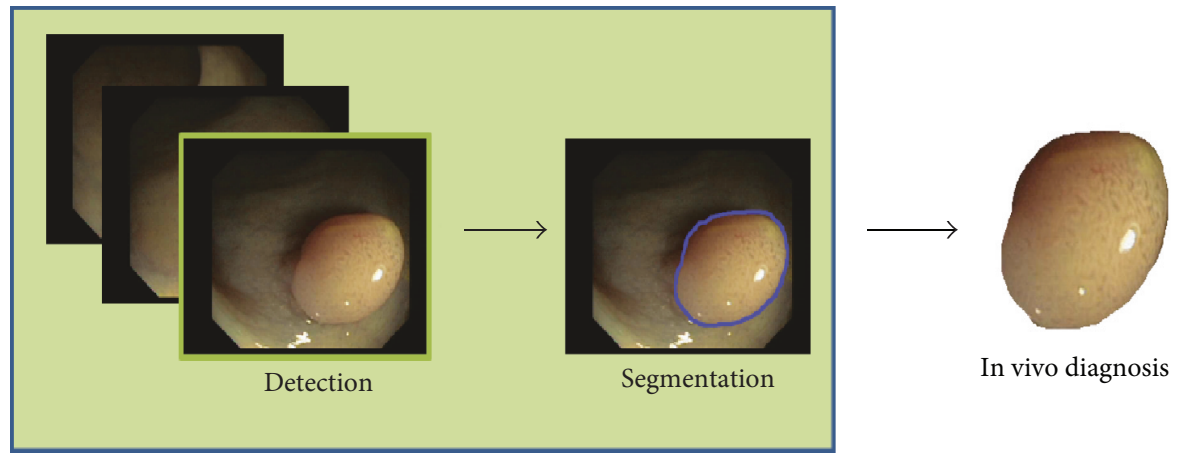

In vivo diagnosis

(b)

Figure 1: (a) Colonoscopy image and corresponding labeling: blue for lumen, red for background (mucosa wall), and green for polyp. (b) Proposed pipeline of a decision support system for colonoscopy.

The development of DSS for colonoscopy has been an active research topic during the last decades. The majority of available works on optical colonoscopy are focused on polyp detection (e.g., see [6-11]), and only few works address the problems of endoluminal scene segmentation.

Endoluminal scene segmentation is of crucial relevance for clinical applications [6, 12-14]. Polyp segmentation is important to define the area covered by a potential lesion that should be carefully inspected and possibly removed by clinicians. Moreover, having a system for accurate in vivo prediction of polyp histology might significantly improve clinical workflow. Lumen segmentation is relevant to help clinicians navigate through the colon during the procedure. Additionally, it can be used to establish quality metrics related to the degree of the colon wall that has been explored, since a weak exploration can lead to polyp overlooking. Finally, specular highlights have proven to be useful in reducing polyp detection false-positive ratio in the context of handcrafted methods [15].

In recent years, convolutional neural networks (CNNs) have become a de facto standard in computer vision, achieving state-of-the-art performance in tasks such as image classification, object detection, and semantic segmentation; and making traditional methods based on handcrafted features obsolete. Two major components in this groundbreaking progress were the availability of increased computational power (GPUs) and the introduction of large labeled datasets $[16,17]$. Despite the additional difficulty of having limited amounts of labeled data, CNNs have successfully been applied to a variety of medical imaging tasks, by resorting to aggressive data augmentation techniques [18, 19]. More precisely, CNNs have excelled at semantic segmentation tasks in medical imaging, such as the EM ISBI 2012 dataset [20], BRATS [21], or MS lesions [22], where the top entries are built on CNNs [18, 19, 23-25]. Surprisingly, to the best of our knowledge, CNNs have not been applied to semantic segmentation of colonoscopy data. We associate this to the lack of large publicly available annotated databases, which are needed in order to train and validate such networks.

In this paper, we aim to overcome this limitation by introducing an extended benchmark of colonoscopy images created from the combination of the two largest public datasets of colonoscopy images $[6,26]$ and by incorporating additional annotations to segment lumen and specular highlights, with the hope of establishing a new strong benchmark for colonoscopy image analysis research. We provide new baselines on this dataset by training standard fully convolutional networks (FCNs) for semantic segmentation [27] and significantly outperforming, without any further postprocessing, prior results in endoluminal scene segmentation.

Therefore, the contributions of this paper are twofold:

(1) Extended benchmark for colonoscopy image segmentation

(2) New state-of-the-art in colonoscopy image segmentation. 
TABLE 1: Summary of prior database content. All frames show at least one polyp.

\begin{tabular}{lccccc}
\hline Database & Number of patients & Number of seq. & Number of frames & Resolution & Annotations \\
\hline CVC-ColonDB & 13 & 13 & 300 & $500 \times 574$ & Polyp, lumen \\
CVC-ClinicDB & 23 & 31 & 612 & $384 \times 288$ & Polyp \\
CVC-EndoSceneStill & 36 & 44 & 912 & $500 \times 574 \& 384 \times 288$ & $\begin{array}{c}\text { Polyp, lumen, background, } \\
\text { specularity, border (void) }\end{array}$ \\
\hline
\end{tabular}

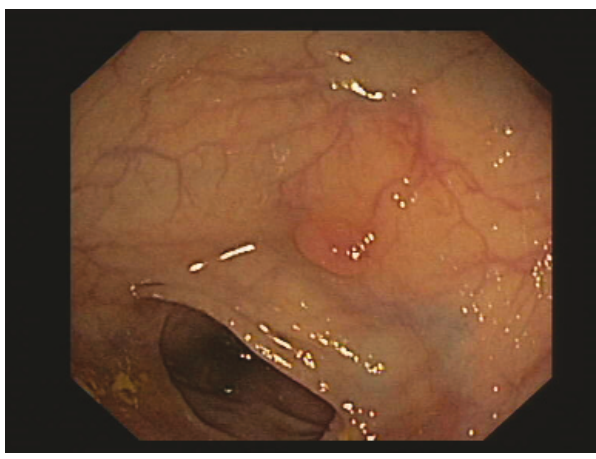

(a)

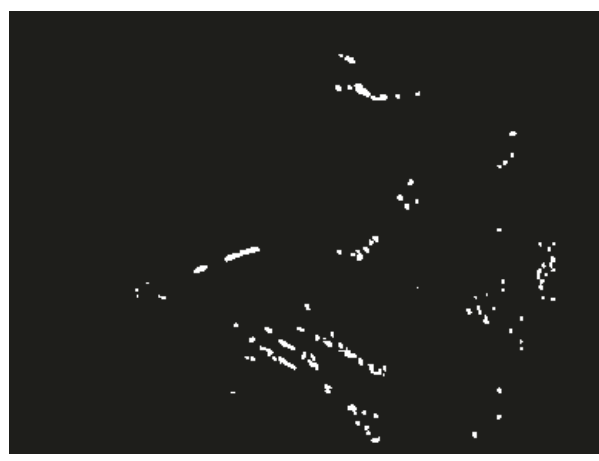

(c)

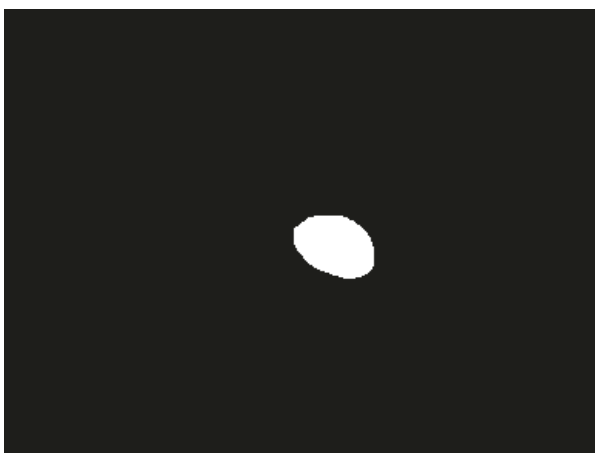

(b)

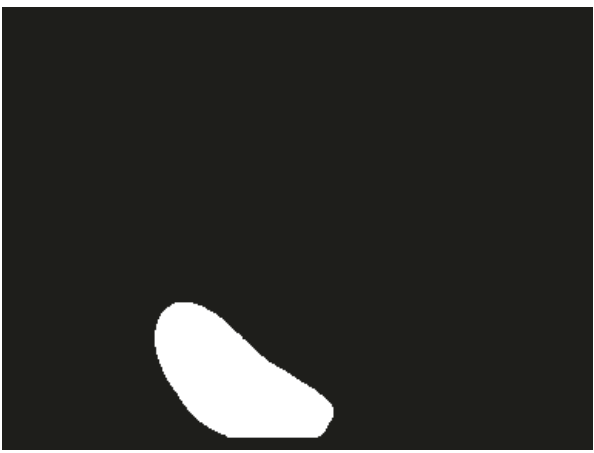

(d)

FIGURE 2: Example of a colonoscopy image and its corresponding ground truth: (a) original image, (b) polyp mask, (c) specular highlights mask, and (d) lumen mask.

The rest of the paper is organized as follows. In Section 2, we present the new extended benchmark, including the introduction of datasets as well as the performance metrics. After that, in Section 3, we introduce the FCN architecture used as baseline for the new endoluminal scene segmentation benchmark. Then, in Section 4, we show qualitative and quantitative experimental results. Finally, Section 5 concludes the paper.

\section{Endoluminal Scene Segmentation Benchmark}

In this section, we describe the endoluminal scene segmentation benchmark, including evaluation metrics.

2.1. Dataset. Inspired by already published benchmarks for polyp detection, proposed within a challenge held in conjunction with MICCAI 2015 (http://endovis.grand-challenge.org)
[28], we introduce a benchmark for endoluminal scene object segmentation.

We combine $C V C$-Colon $D B$ and $C V C$-Clinic $D B$ into a new dataset (CVC-EndoSceneStill) composed of 912 images obtained from 44 video sequences acquired from 36 patients.

(i) CVC-ColonDB contains 300 images with associated polyp masks obtained from 13 polyp video sequences acquired from 13 patients.

(ii) CVC-ClinicDB contains 612 images with associated polyp and background (here, mucosa and lumen) segmentation masks obtained from 31 polyp video sequences acquired from 23 patients.

We extend the old annotations to account for lumen, specular highlights with new hand-made pixel-wise annotations, and we define a void class for black borders present in each frame. In the new annotations, background only 
contains mucosa (intestinal wall). Please refer to Table 1 for dataset details and to Figure 2 for a dataset sample.

We split the resulting dataset into three sets: training, validation, and test containing $60 \%, 20 \%$, and $20 \%$ images, respectively. We impose the constraint that one patient cannot be in different sets. As a result, the final training set contains 20 patients and 547 frames, the validation set contains 8 patients and 183 frames, and the test set contains 8 patients and 182 frames. The dataset is publicly available (http://www.cvc.uab.es/CVC-Colon/ index.php/databases/cvc-endoscenestill/).

2.2. Metrics. We use Intersection over Union (IoU), also known as Jaccard index, and per pixel accuracy as segmentation metrics. These metrics are commonly used in medical image segmentation tasks $[29,30]$.

We compute the mean of per class IoU. Each per class IoU is computed over a validation/test set according to the following formula:

$$
\operatorname{IoU}(\operatorname{PR}(\text { class }), \mathrm{GT}(\text { class }))=\frac{\mid \operatorname{PR}(\text { class }) \cap \mathrm{GT}(\text { class }) \mid}{\mid \operatorname{PR}(\text { class }) \cup \mathrm{GT}(\text { class }) \mid},
$$

where PR represents the binary mask produced by the segmentation method, GT represents the ground truth mask, $\cap$ represents set intersection, and $\cup$ represents set union.

We compute the mean global accuracy for each set as follows:

$$
\operatorname{Acc}(\mathrm{PR}, \mathrm{GT})=\frac{\# \mathrm{TP}}{\# \text { pixels }},
$$

where TP represents the number of true positives.

Notably, this new benchmark might as well be used for the relevant task of polyp localization. In that case, we follow Pascal VOC challenge metrics [31] and determine that a polyp is localized if it has a high overlap degree with its associated ground truth, namely,

$$
\mathrm{IoU}(\mathrm{PR}(\text { polyp }), \mathrm{GT}(\text { polyp }))>0.5 \text {, }
$$

where the metric is computed for each polyp independently and averaged per set to give a final score.

\section{Baseline}

CNNs are a standard architecture used for tasks, where a single prediction per input is expected (e.g., image classification). Such architectures capture hierarchical representations of the input data by stacking blocks of convolutional, nonlinearity, and pooling layers on top of each other. Convolutional layers extract local features. Nonlinearity layers allow deep networks to learn nonlinear mappings of the input data. Pooling layers reduce the spatial resolution of the representation maps by aggregating local statistics.

FCNs $[19,27]$ were introduced in the computer vision and medical imaging communities in the context of semantic segmentation. FCNs naturally extend CNNs to tackle per pixel prediction problems, by adding upsampling layers to recover the spatial resolution of the input at the output layer. As a consequence, FCNs can process images of arbitrary size.
In order to compensate for the resolution loss induced by pooling layers, FCNs introduce skip connections between their downsampling and upsampling paths. Skip connections help the upsampling path recover fine-grained information from the downsampling layers.

We implemented FCN8 architecture from [27] and trained the network by means of stochastic gradient descent with the rmsprop adaptive learning rate [32]. The validation split is used to early stop the training; we monitor mean IoU for validation set and use patience of 50 . We used a minibatch size of 10 images. The input image is normalized in the range $0-1$. We randomly crop the training images to $224 \times 224$ pixels. As regularization, we use dropout [33] of 0.5 , as mentioned in the paper [27]. We do not use any weight decay.

As described in Section 2.1, colonoscopy images have a black border that we consider as a void class. Void classes do not influence the computation of the loss nor the metrics of any set, since the pixels marked as void class are ignored. As the number of pixels per class is unbalanced, in some experiments, we apply the median frequency balancing of [34].

During training, we experiment with data augmentation techniques such as random cropping, rotations, zooming, and sharing and elastic transformations.

\section{Experimental Results}

In this section, we report semantic segmentation and polyp localization results on the new benchmark.

4.1. Endoluminal Scene Semantic Segmentation. In this section, we first analyze the influence of different data augmentation techniques. Second, we evaluate the effect of having different numbers of endoluminal classes on polyp segmentation results. Finally, we compare our results with previously published methods.

4.1.1. Influence of Data Augmentation. Table 2 presents an analysis on the influence of different data augmentation techniques and their impact on the validation performance. We evaluate random zoom from 0.9 to 1.1 , rotations from 0 to 180 degrees, shearing from 0 to 0.4 , and warping with $\sigma$ ranging from 0 to 10 . Finally, we evaluate the combination of all the data augmentation techniques.

As shown in the table, polyps significantly benefit from all data augmentation methods, in particular, from warping. Note that warping applies small elastic deformation locally, accounting for many realistic variations in the polyp shape. Rotation and zoom also have a strong positive impact on the polyp segmentation performance. It goes without saying that such transformations are the least aggressive ones, since they do not alter the polyp appearance. Shearing is most likely the most aggressive transformation, since it changes the polyp appearance and might, in some cases, result in unrealistic deformations.

While for lumen it is difficult to draw any strong conclusions, it looks like zooming and warping slightly deteriorate the performance, whereas shearing and rotation slightly improve it. As for specular highlights, all 
TABLE 2: FCN8 endoluminal scene semantic segmentation results for different data augmentation techniques. The results are reported on validation set.

\begin{tabular}{lcccccc}
\hline Data augmentation & IoU background & IoU polyp & IoU lumen & IoU spec. & IoU mean & Acc mean \\
\hline None & 88.93 & 44.45 & 54.02 & 25.54 & 57.88 & 92.48 \\
Zoom & 89.89 & 52.73 & 51.15 & 37.10 & 57.72 & 90.72 \\
Warp & 90.00 & 54.00 & 49.69 & $\mathbf{3 7 . 2 7}$ & 58.97 & 90.93 \\
Shear & 89.60 & 46.61 & 54.27 & 36.86 & 56.83 & 90.49 \\
Rotation & 90.52 & 52.83 & $\mathbf{5 6 . 3 9}$ & 35.81 & 58.89 & 91.38 \\
Combination & $\mathbf{9 2 . 6 2}$ & $\mathbf{5 4 . 8 2}$ & 55.08 & 35.75 & $\mathbf{5 9 . 5 7}$ & $\mathbf{9 3 . 0 2}$ \\
\hline
\end{tabular}

TABLE 3: FCN8 endoluminal scene semantic segmentation results for different numbers of classes. The results are reported on validation set. In all cases, we selected the model that provided best validation results (with or without class balancing).

\begin{tabular}{lcccccc}
\hline Number of classes & IoU background & IoU polyp & IoU lumen & IoU spec. & IoU mean & Acc mean \\
\hline 4 & 92.07 & 39.37 & $\mathbf{5 9 . 5 5}$ & $\mathbf{4 0 . 5 2}$ & 57.88 & 92.48 \\
3 & 92.19 & 50.70 & 56.48 & - & 66.46 & 92.82 \\
2 & $\mathbf{9 6 . 6 3}$ & $\mathbf{5 6 . 0 7}$ & - & - & $\mathbf{7 6 . 3 5}$ & $\mathbf{9 6 . 7 7}$ \\
\hline
\end{tabular}

TABLE 4: Results on the test set: FCN8 with respect to previously published methods.

\begin{tabular}{|c|c|c|c|c|c|c|c|}
\hline & Data augmentation & IoU background & IoU polyp & IoU lumen & IoU spec. & IoU mean & $\overline{\text { Acc mean }}$ \\
\hline \multicolumn{8}{|c|}{ FCN8 performance } \\
\hline 4 classes & None & 86.36 & 38.51 & 43.97 & 32.98 & 50.46 & 87.40 \\
\hline 3 classes & None & 84.66 & 47.55 & 36.93 & - & 56.38 & 86.08 \\
\hline 2 classes & None & 94.62 & 50.85 & - & - & 72.74 & 94.91 \\
\hline 4 classes & Combination & 88.81 & 51.60 & 41.21 & 38.87 & 55.13 & 89.69 \\
\hline \multicolumn{8}{|c|}{ State-of-the-art methods } \\
\hline$[12,13,15]$ & - & 73.93 & 22.13 & 23.82 & 44.86 & 41.19 & 75.58 \\
\hline
\end{tabular}

the data augmentation techniques that we tested significantly boost the segmentation results. Finally, background (mucosa) shows only slight improvement when incorporating data augmentations. This is not surprising; given its predominance throughout the data, it could be even considered background.

Overall, combining all the discussed data augmentation techniques leads to better results in terms of mean IoU and mean global accuracy. More precisely, we increase the mean IoU by $4.51 \%$ and the global mean accuracy by $1.52 \%$.

4.1.2. Influence of the Number of Classes. Table 3 presents endoluminal scene semantic segmentation results for different numbers of classes. As shown in the table, using more underrepresented classes such as lumen or specular highlights makes the optimization problem more difficult. As expected and contrary to handcrafted segmentation methods, when considering polyp segmentation, deep learning-based approaches do not suffer from specular highlights, showing the robustness of the learnt features towards saturation zones in colonoscopy images.

Best results for polyp segmentation are obtained in the 2 -class scenario (polyp versus background). However, segmenting lumen is a relevant clinical problem as mentioned in Section 1. Results achieved in the 3-class scenario are very encouraging, with a IoU higher than $50 \%$ for both polyp and lumen classes.

4.1.3. Comparison to State-of-the-Art. Finally, we evaluate the FCN model on the test set. We compare our results to the combination of previously published handcrafted methods: map-based method (1) for polyp segmentation and [12] a watershed-based method (2) for lumen segmentation and [15] (3) for specular highlights segmentation.

The segmentation results on the test set are reported in Table 4 and show a clear improvement of FCN8 over previously published methods. The following improvements can be observed when comparing previously published methods to the 4-class FCN8 model trained with data augmentation: $15 \%$ in IoU for background (mucosa), $29 \%$ in IoU for polyps, $18 \%$ in IoU for lumen, $14 \%$ in mean IoU, and $14 \%$ in mean accuracy. FCN8 is still outperformed by traditional methods when it comes to specular highlight class. However, it is important to note that specular highlight class is used by handcrafted methods to reduce false-positive ratio of polyp detection, and from our analysis, it looks like the FCN model is able to segment well polyps even when ignoring this class. For example, the best mean IoU of $72.74 \%$ and mean 


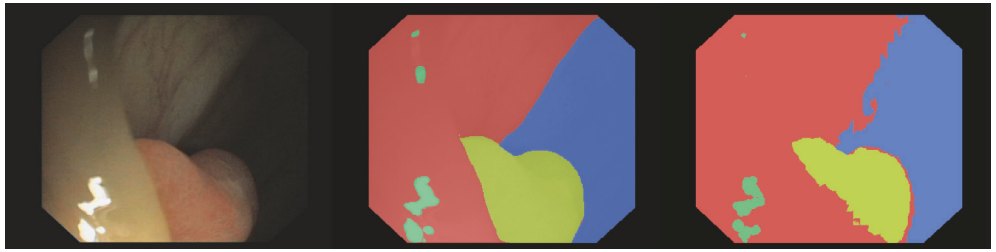

(a)

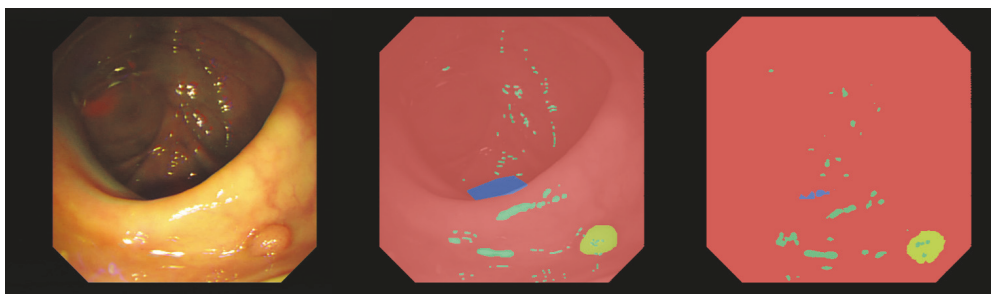

(b)

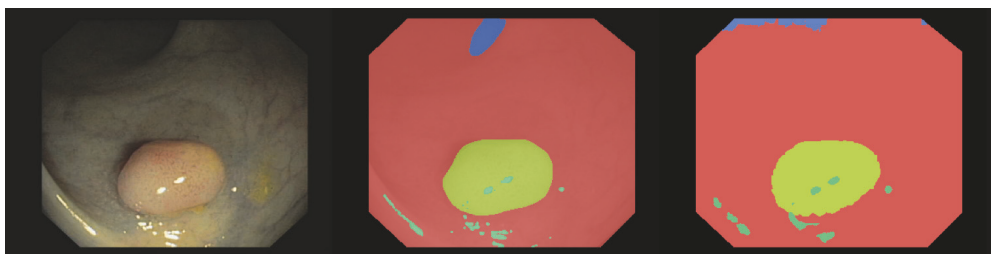

(c)

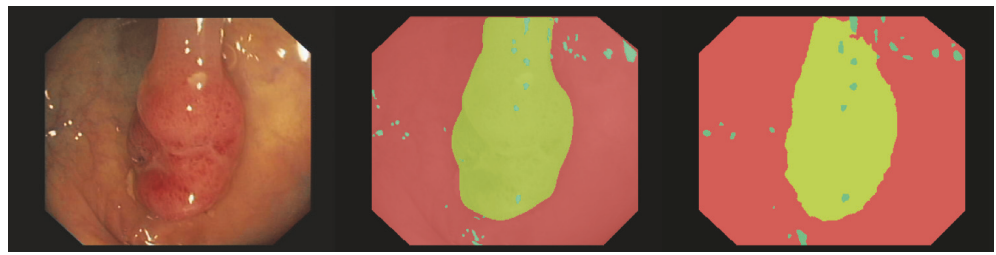

(d)

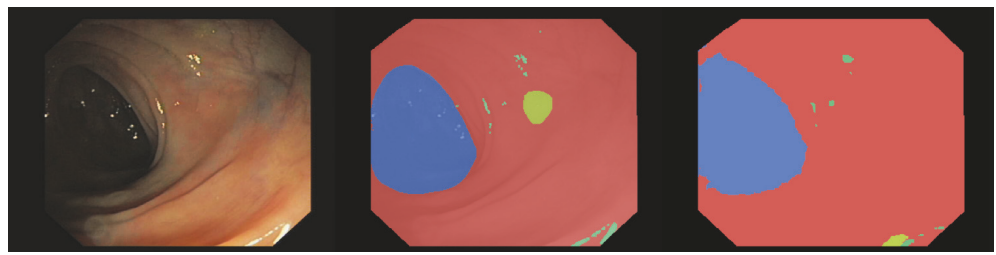

(e)

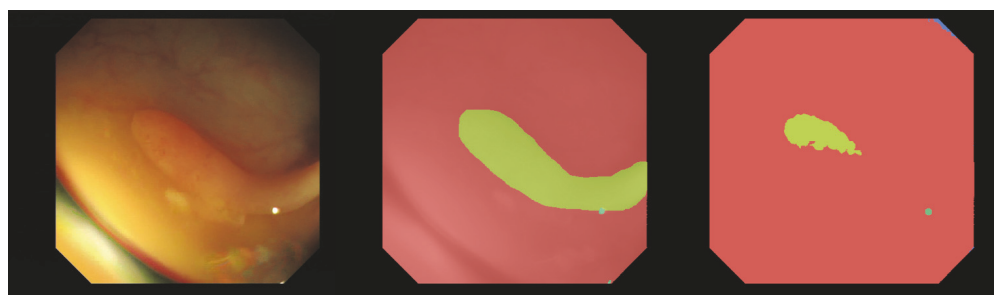

(f)

FIgURE 3: Examples of predictions for 4-class FCN8 model. Each subfigure represents a single frame, a ground truth annotation, and a prediction image. We use the following color-coding in the annotations: red for background (mucosa), blue for lumen, yellow for polyp, and green for specularity. (a), (b), (c), (d) show correct polyp segmentation, whereas (e), (d) show incorrect polyp segmentation.

accuracy of $94.91 \%$ are obtained by the 2 -class model without additional data augmentation.

Figure 3 shows qualitative results of the 4-class FCN8 model trained with data augmentation. From left to right, each row shows a colonoscopy frame, followed by the corresponding ground truth annotation and FCN8 prediction. Rows 1 to 4 show correct segmentation masks, with very clean polyp segmentation. Rows 5 and 6 show failure modes 


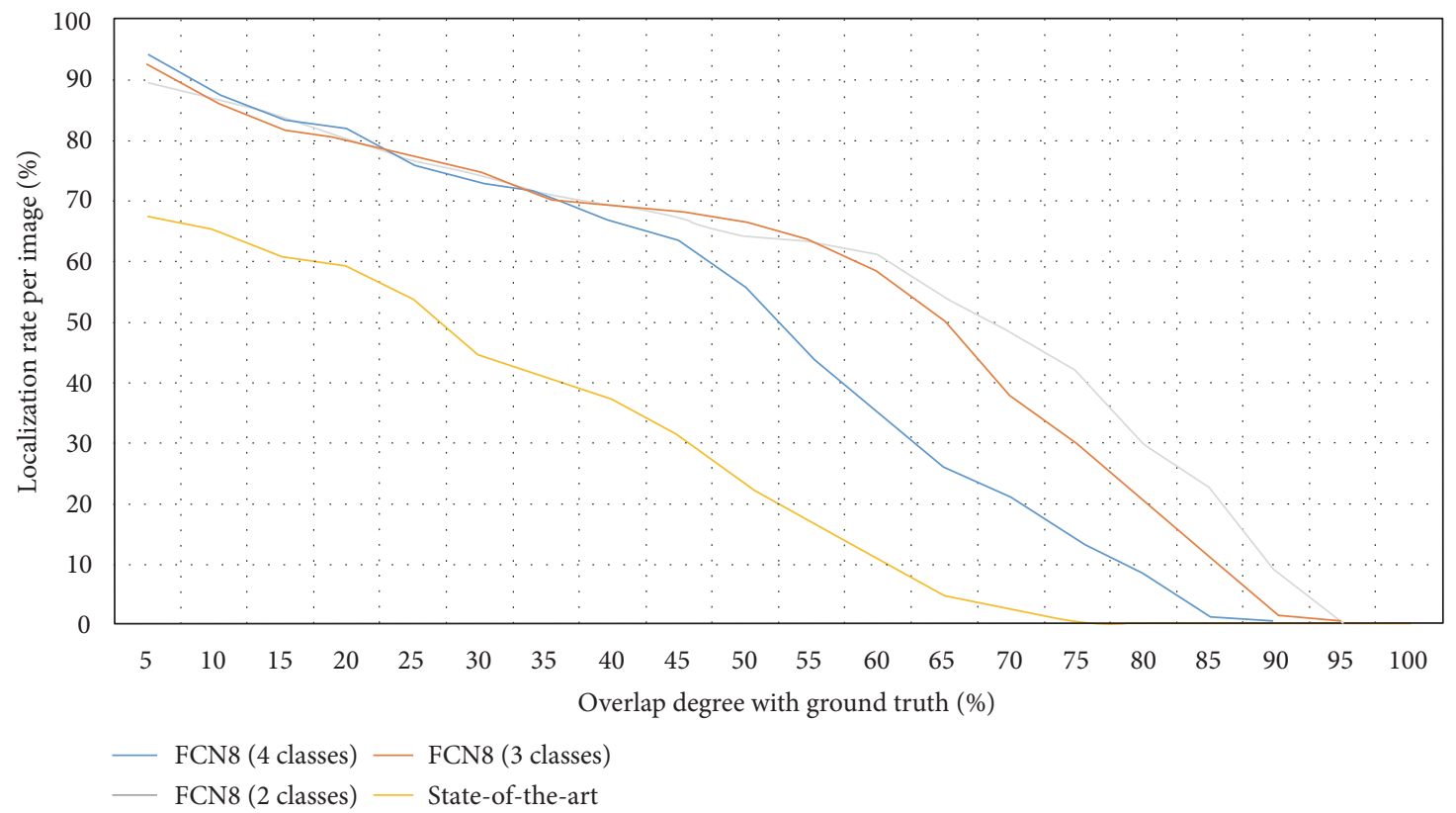

FIGURE 4: Localization rate of polyps as a function of IoU. The $x$-axis represents the degree of overlap between ground truth and model prediction. The $y$-axis represents the percentage of correctly localized polyps. Different color plots represent different models: FCN8 with 4 classes, FCN8 with 3 classes, and FCN8 with 2 classes and previously published method [13] (referred to as state-of-the-art in the plot).

TABle 5: Summary of processing times achieved by the different methods studied in the paper. FCN results are the same for all four classes considered as segmentation of the four classes is done at the same time*.

\begin{tabular}{lcccc}
\hline Method & Polyp & Lumen & $\begin{array}{c}\text { Specular } \\
\text { highlights }\end{array}$ & Background \\
\hline FCN & $88 \mathrm{~ms}^{*}$ & $88 \mathrm{~ms}^{*}$ & $88 \mathrm{~ms}^{*}$ & $88 \mathrm{~ms}^{*}$ \\
State-of-the-art & $10000 \mathrm{~ms}$ & $8000 \mathrm{~ms}$ & $5000 \mathrm{~ms}$ & $23000 \mathrm{~ms}$ \\
\hline
\end{tabular}

of the model, where polyps have been missed or undersegmented. In row 5 , the small polyp is missed by our segmentation method while, in row 6, the polyp is undersegmented. All cases exhibit decent lumen segmentation and good background (mucosa) segmentation.

4.2. Polyp Localization. Endoluminal scene segmentation can be seen as a proxy to proper polyp detection in a colonoscopy video. In order to understand how well suited FCNs are to localize polyps, we perform a last experiment. In this experiment, we compute the polyp localization rate as a function of IoU between the model prediction and the ground truth. We can compute this IoU per frame, since our dataset contains a maximum of one polyp per image. This analysis describes the ability of a given method to cope with polyp appearance variability and stability on polyp localization.

The localization results are presented in Figure 4 and show a significant improvement when comparing FCN8 variants to the previously published method [13]. For example, when considering a correct polyp localization to have at least $50 \%$ IoU, we observe an increase of $40 \%$ in the polyp localization rate. As a general trend, we observe that architectures trained using a fewer number of classes achieve a higher IoU, though the polyp localization difference starts to be more visible when really high overlapping degrees are imposed. Finally, as one would expect, we observe that the architectures that show better results in polyp segmentation are the ones that show better results in polyp localization.

4.3. Towards Clinical Applicability. Sections 4.1 .3 and 4.2 presented results of a comparative study between FCNs and previous state-of-the-art of endoluminal scene object segmentation in colonoscopy images. As mentioned in Section 1, we foresee several clinical applications, which can be built from the results of endoluminal scene segmentation. However, in order to be deployed in the exploration room, they must comply with real-time constraints apart from offering a good segmentation performance. In this case and considering videos recorded at 25 frames per second, a DSS should not take more than $40 \mathrm{~ms}$ to process an image in order not to delay the procedure.

Considering this, we have computed processing times for each of the approaches studied in this paper. Results are presented in Table 5.

As shown in the table, none of the presented approaches currently meet real-time constraints. Running the FCN8 inference on an NVIDIA Titan X GPU takes $88 \mathrm{~ms}$ per frame. Note that this could easily be addressed by taking advantage of recent research on model compression [35] by applying fancier FCN architectures that encourage feature reuse [36]. Alternatively, we could exploit the temporal component and build more sophisticated architectures that would take advantage of the similarities among consecutive frames. 
Clearly, handcrafted methods take much longer to process one image. Moreover, they need to apply different methods to segment each class of interest, making them less clinically useful. Note that this is not the case for FCN-like architectures.

Despite computational constraints, FCNs' superior performance could lead to more reliable and impactful computer-assisted clinical applications, since they offer both a better performance and computational efficiency.

\section{Conclusions}

In this paper, we have introduced an extended benchmark for endoluminal scene semantic segmentation. The benchmark includes extended annotations of polyps, background (mucosa), lumen, and specular highlights. The dataset provides the standard training, validation, and test splits for machine learning practitioners and will be publicly available upon paper acceptance. Moreover, standard metrics for the comparison have been defined, with the hope to speed up the research in the endoluminal scene segmentation area.

Together with the dataset, we provided new baselines based on fully convolutional networks, which outperformed by a large margin previously published results, without any further postprocessing. We extended the proposed pipeline and used it as proxy to perform polyp detection. Due to the lack of nonpolyp frames in the dataset, we reformulated the task as polyp localization. Once again, we highlighted the superiority of deep learning-based models over traditional handcrafted approaches. As expected and contrary to handcrafted segmentation methods, when considering polyp segmentation, deep learning-based approaches do not suffer from specular highlights, showing the robustness of the learnt features towards saturation zones in colonoscopy images. Moreover, given that FCN not only excels in terms of performance but also allows for nearly real-time processing, it has a great potential to be included in future DSS for colonoscopy.

Knowing the potential of deep learning techniques, efforts in the medical imaging community should be devoted to gather larger labeled datasets as well as designing deep learning architectures that would be better suited to deal with colonoscopy data. This paper pretends to make a first step towards novel and more accurate DSS by making all code and data publicly available, paving the road for more researchers to contribute to the endoluminal scene segmentation domain.

\section{Conflicts of Interest}

The authors declare that they have no conflicts of interest.

\section{Acknowledgments}

The authors would like to thank the developers of Theano [37] and Keras [38]. The authors acknowledge the support of the following agencies for research funding and computing support: Imagia Inc.; Spanish government through funded Project AC/DC TRA2014-57088-C2-1-R and iVENDIS (DPI2015-65286-R); SGR Projects 2014-SGR-1506, 2014-SGR-1470, and 2014-SGR-135; CERCA Programme/ Generalitat de Catalunya; and TECNIOspring-FP7-ACCI grant, FSEED, and NVIDIA Corporation for the generous support in the form of different GPU hardware units.

\section{References}

[1] Society, A.C, Colorectal cancer, 2016.

[2] A. Leufkens, M. Van Oijen, F. P. Vleggaar, and P. D. Siersema, "Factors inuencing the miss rate of polyps in a back-to-back colonoscopy study," Endoscopy, vol. 44, no. 5, pp. 470-475, 2012.

[3] H. Machida, Y. Sano, Y. Hamamoto et al., "Narrow-band imaging in the diagnosis of colorectal mucosal lesions: a pilot study," Endoscopy, vol. 36, no. 12, pp. 1094-1098, 2004.

[4] M. Bruno, "Magnification endoscopy, high resolution endoscopy, and chromoscopy; towards a better optical diagnosis," Gut, vol. 52, Supplement 4, pp. iv7-iv11, 2003.

[5] H. K. Roy, M. J. Goldberg, S. Bajaj, and V. Backman, "Colonoscopic optical biopsy: bridging technological advances to clinical practice," Gastroenterology, vol. 140, no. 7, p. 1863, 2011.

[6] J. Bernal, F. J. Sánchez, G. Fernández-Esparrach, D. Gil, C. Rodríguez, and F. Vilariño, "WM-DOVA maps for accurate polyp highlighting in colonoscopy: validation vs. saliency maps from physicians," Computerized Medical Imaging and Graphics, vol. 43, pp. 99-111, 2015.

[7] S. Gross, T. Stehle, A. Behrens et al., "A comparison of blood vessel features and local binary patterns for colorectal polyp classification," in SPIE Medical Imaging, article 72602Q, International Society for Optics and Photonics, 2009.

[8] S. Y. Park and D. Sargent, "Colonoscopic polyp detection using convolutional neural networks," in SPIE Medical Imaging, article 978528, International Society for Optics and Photonics, 2016.

[9] E. Ribeiro, A. Uhl, and M. Häfner, "Colonic polyp classification with convolutional neural networks," in 2016 IEEE 29th International Symposium on Computer-Based Medical Systems (CBMS), pp. 253-258, Dublin, 2016.

[10] J. Silva, A. Histace, O. Romain, X. Dray, and B. Granado, "Toward embedded detection of polyps in wce images for early diagnosis of colorectal cancer," International Journal of Computer Assisted Radiology and Surgery, vol. 9, no. 2, pp. 283-293, 2014.

[11] N. Tajbakhsh, S. Gurudu, and J. Liang, "Automated polyp detection in colonoscopy videos using shape and context information," IEEE Transactions on Medical Imaging, vol. 35, no. 2, pp. 630-644, 2015.

[12] J. Bernal, D. Gil, C. Sánchez, and F. J. Sánchez, "Discarding non informative regions for efficient colonoscopy image analysis," in International Workshop on Computer-Assisted and Robotic Endoscopy, pp. 1-10, Springer, 2014.

[13] J. Bernal, J. M. Núñez, F. J. Sánchez, and F. Vilariño, "Polyp segmentation method in colonoscopy videos by means of MSADOVA energy maps calculation," in MICCAI 2014 Workshop on Clinical Image-Based Procedures, pp. 41-49, Springer, 2014.

[14] J. M. Núñez, J. Bernal, M. Ferrer, and F. Vilariño, "Impact of keypoint detection on graph-based characterization of blood vessels in colonoscopy videos," in International Workshop on Computer-Assisted and Robotic Endoscopy, pp. 22-33, Springer, 2014. 
[15] J. Bernal, J. Sánchez, and F. Vilarino, "Impact of image preprocessing methods on polyp localization in colonoscopy frames," in 2013 35th Annual International Conference of the IEEE Engineering in Medicine and Biology Society (EMBC), pp. 7350-7354, Osaka, 2013.

[16] J. Deng, W. Dong, R. Socher, L. J. Li, K. Li, and L. Fei-Fei, "ImageNet: a large-scale hierarchical image database," CVPR09, 2009.

[17] T. Y. Lin, M. Maire, S. Belongie et al., "Microsoft coco: common objects in context," in European Conference on Computer Vision (ECCV), Zurich, 2014.

[18] M. Drozdzal, E. Vorontsov, G. Chartrand, S. Kadoury, and C. $\mathrm{Pal}$, "The importance of skip connections in biomedical image segmentation," 2016, http://arxiv.org/abs/1608.04117.

[19] O. Ronneberger, P. Fischer, and T. Brox, "U-net: convolutional networks for biomedical image segmentation," in International Conference on Medical Image Computing and ComputerAssisted Intervention, pp. 234-241, Springer, Cham, 2015.

[20] I. Arganda-Carreras, S. C. Turaga, D. R. Berger et al., "Crowdsourcing the creation of image segmentation algorithms for connectomics," Frontiers in Neuroanatomy, vol. 9, p. 142, 2015.

[21] B. Menze, A. Jakab, S. Bauer et al., "The multimodal brain tumor image segmentation benchmark (BRATS)," IEEE Transactions on Medical Imaging, vol. 34, no. 10, pp. 19932024, 2014.

[22] M. Styner, J. Lee, B. Chin et al., "3d segmentation in the clinic: a grand challenge ii: Ms lesion segmentation," Midas Journal, vol. 2008, pp. 1-6, 2008.

[23] T. Brosch, L. Y. W. Tang, Y. Yoo, D. K. Li, A. Traboulsee, and R. Tam, "Deep 3d convolutional encoder networks with shortcuts for multiscale feature integration applied to multiple sclerosis lesion segmentation," IEEE Transactions on Medical Imaging, vol. 35, no. 5, pp. 1229-1239, 2016.

[24] H. Chen, X. Qi, J. Cheng, and P. A. Heng, "Deep contextual networks for neuronal structure segmentation," in Proceedings of the 13th AAAI Conference on Artificial Intelligence, pp. 1167-1173, Phoenix, Arizona, USA, February 2016.

[25] M. Havaei, A. Davy, D. Warde-Farley et al., "Brain tumor segmentation with deep neural networks," 2015, http://arxiv. org/abs/1505.03540.

[26] J. Bernal, J. Sánchez, and F. Vilarino, "Towards automatic polyp detection with a polyp appearance model," Pattern Recognition, vol. 45, no. 9, pp. 3166-3182, 2012.

[27] J. Long, E. Shelhamer, and T. Darrell, "Fully convolutional networks for semantic segmentation," Proceedings of the IEEE Conference on Computer Vision and Pattern Recognition, pp. 3431-3440, Boston, MA, 2015.

[28] J. Bernal, N. Tajbakhsh, F. J. Sanchez et al., "Comparative validation of polyp detection methods in video colonoscopy: results from the miccai 2015 endoscopic vision challenge," IEEE Transactions on Medical Imaging, vol. 36, no. 6, pp. 1231-1249, 2017.

[29] K. H. Cha, L. Hadjiiski, R. K. Samala, H. P. Chan, E. M. Caoili, and R. H. Cohan, "Urinary bladder segmentation in ct urography using deep-learning convolutional neural network and level sets," Medical Physics, vol. 43, no. 4, pp. 1882-1896, 2016.

[30] M. Prastawa, E. Bullitt, S. Ho, and G. Gerig, "A brain tumor segmentation framework based on outlier detection," Medical Image Analysis, vol. 8, no. 3, pp. 275-283, 2004.
[31] M. Everingham, L. Van Gool, C. K. I. Williams, J. Winn, and A. Zisserman, "The pascal visual object classes (voc) challenge," International Journal of Computer Vision, vol. 88, no. 2, pp. 303-338, 2010.

[32] T. Tieleman and G. Hinton, "rmsprop adaptive learning," COURSERA: Neural Networks for Machine Learning, 2012.

[33] N. Srivastava, G. Hinton, A. Krizhevsky, I. Sutskever, and R. Salakhutdinov, "Dropout: a simple way to prevent neural networks from overfitting," Journal of Machine Learning Research, vol. 15, no. 1, pp. 1929-1958, 2014.

[34] D. Eigen and R. Fergus, "Predicting depth, surface normals and semantic labels with a common multi-scale convolutional architecture," 2014, http://arxiv.org/abs/1411.4734.

[35] A. Romero, N. Ballas, S. E. Kahou, A. Chassang, C. Gatta, and Y. Bengio, "Fitnets: hints for thin deep nets," in Proceedings of ICLR, 2015.

[36] G. Huang, Z. Liu, K. Q. Weinberger, and L. van der Maaten, "Densely connected convolutional networks," 2016, http:// arxiv.org/abs/1608.06993.

[37] Theano Development Team, "Theano: a python framework for fast computation of mathematical expressions," 2016, http://arxiv.org/abs/1605.02688.

[38] F. Chollet, "Keras," 2015, https://github.com/fchollet/keras. 


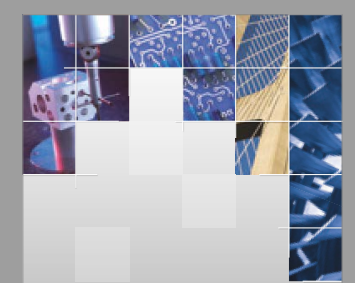

\section{Enfincering}
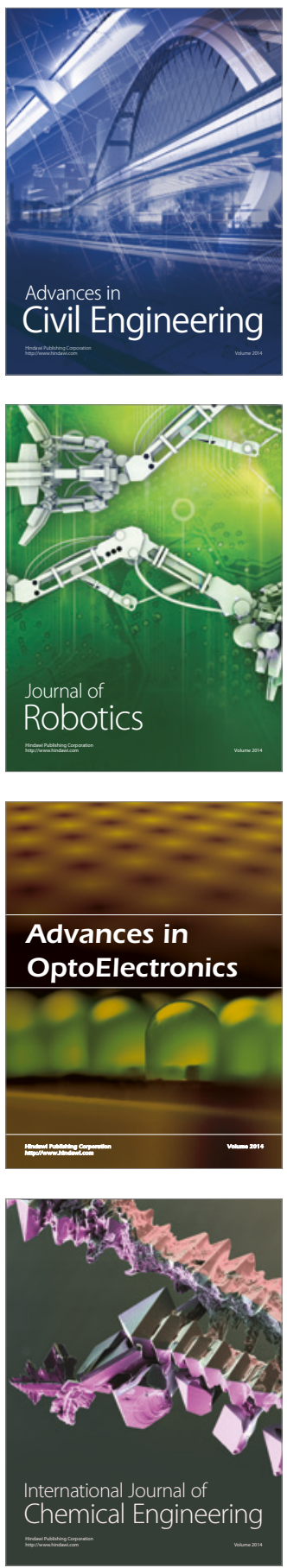

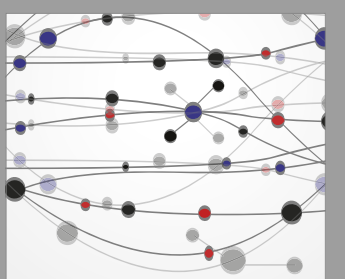

The Scientific World Journal

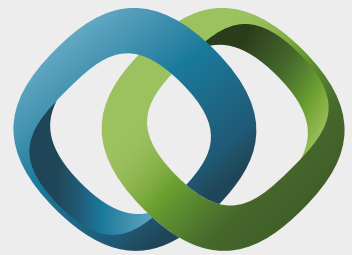

\section{Hindawi}

Submit your manuscripts at

https://www.hindawi.com
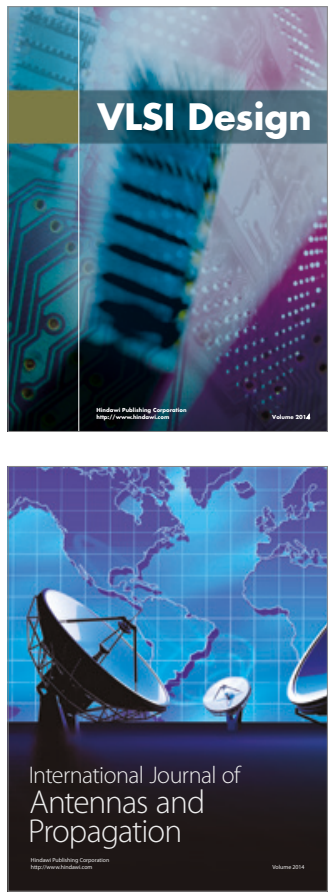

\section{Rotating}

Machinery
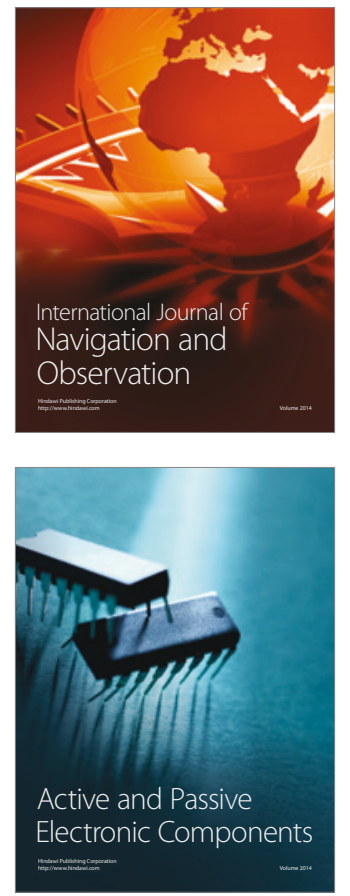
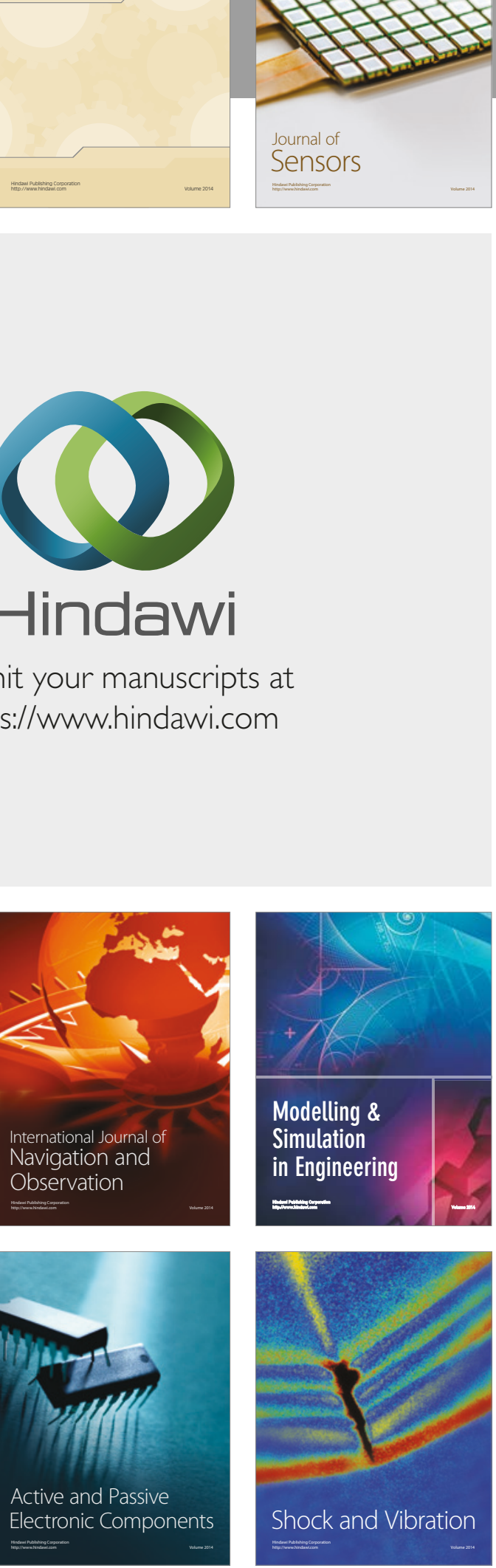
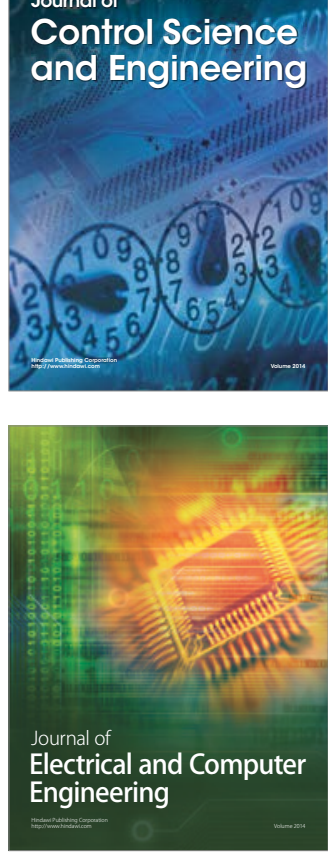

Distributed

Journal of

Control Science

and Engineering
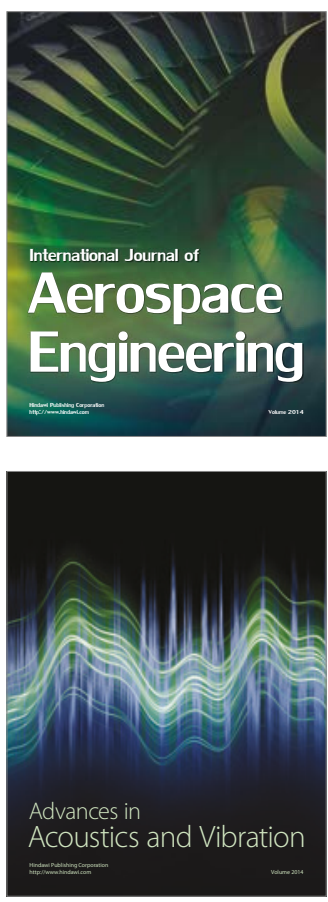

Sensor Networks 